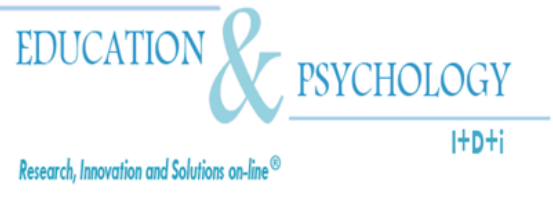

\title{
Impacto de un curso sobre maltrato entre iguales en el currículum universitario del profesorado ${ }^{1}$
}

\author{
Juan L. Benítez Muñoz, Ana García Berbén, \\ María Fernández Cabezas
}

Departamento de Psicología Evolutiva y de la Educación

Universidad de Granada

\section{España}

Juan L. Benítez Muñoz. Facultad de Ciencias de la Educación. Dpto. de Psicología Evolutiva y de la educación. Campus Cartuja s/n, 18071. Granada - España. E-mail: jlbenit@ugr.es

(C) Education \& Psychology I+D+i and Editorial EOS (Spain)

${ }^{1}$ El artículo se desarrolla dentro del Proyecto I+D+I del Ministerios de Ciencia y Tecnología con código SEJ2005-04644. 


\section{Resumen}

Introducción. Aunque el maltrato entre alumnos afecta directamente al profesorado, la formación de estos profesionales para hacer frente al fenómeno es escasa. Una de las posibles vías de intervención para evitar esta situación es la inclusión de contenidos de formación específicos sobre maltrato entre iguales en el currículum universitario. Se presenta un trabajo que muestra el impacto de un curso de formación sobre malos tratos entre iguales sobre los conocimientos, percepciones y actitudes del profesorado en formación.

Método. Los participantes $(\mathrm{n}=199)$ son profesores en formación de Educación Infantil, Primaria y Secundaria. Para la recogida de datos se utiliza el Cuestionario sobre maltrato entre iguales en la escuela (2002) adaptado al español para esta investigación. Ésta se realiza siguiendo un diseño cuasi-experimental pre-post de dos grupos no equivalentes.

Resultados. Los resultados obtenidos indican mejoras estadísticamente significativas entre los miembros del grupo experimental relacionadas con la caracterización del fenómeno, identificación de agentes implicados, caracterización de víctimas y agresores, elección de estrategias de enfrentamiento al maltrato, así como una mejora en la percepción de la auto-eficacia para afrontar con garantías el tratamiento del problema.

Discusión y conclusiones. Los resultados obtenidos, acordes a otras investigaciones realizadas, permiten afirmar que los miembros del grupo experimental han modificado y mejorado los conocimientos y percepciones sobre el maltrato entre iguales. La formación específica tiene un impacto positivo y pone de manifiesto la necesidad de introducir contenidos específicos en los planes de estudio del profesorado que permitan al alumnado conocer problemas educativos.

Palabras clave. Maltrato entre iguales, profesorado, intervención, currículum universitario.

Recibido: 04/09/08 Aceptación inicial: 04/09/08 Aceptación final: 14/10/08 


\begin{abstract}
Introduction. Although bullying directly affects teachers their formation for facing it is scarce. One of the possible intervention ways for avoiding this situation is to include specific contents about bullying within initial university curriculum. We present a work that shows the impact of specific training on trainee teachers' knowledge, perceptions and attitudes towards this problem.

Method. The participants $(\mathrm{n}=199)$ are trainee teachers (university students) of Pre-School, Elementary and Secondary Education distributed in experimental and control groups. For collecting data was used the Questionnaire about bullying in the schools (Nicolaides, Toda \& Smith, 2002) adapted to Spanish for this research. A quasi-experimental pre-post design with non-equivalent groups is used.

Results. The results point out significant statistical improvement among experimental group members related with characterization of the phenomenon, detection of involved agents, victims and aggressors characterization, coping strategies for tackling bullying as well as an improvement of self-efficacy for facing bullying with guarantee.

Discussion and conclusions. The results similar to other researches allows to affirm that experimental group members have modified and improved their knowledge and perceptions about bullying. Specific training has a positive impact and advice about the necessity of including specific contents about scholar problems into the university teachers' curriculum those allow them to know about such phenomenons.
\end{abstract}

Keywords: bullying, teachers, intervention, university curriculum

Received: 09/04/08 Initial Acceptance: 09/04/08 Final Acceptance: 10/14/08 


\section{Introducción}

Cuando se utiliza el término maltrato entre iguales nos referimos a un fenómeno de carácter físico, verbal, psicológico y/o relacional, que surge con la intención de dañar a otra persona, que es frecuente y duradero y en el que existe una asimetría de poder entre víctima y agresor (Cerezo, 2006; Del Barrio, Gutiérrez, Van der Meulen, Barrios y Granizo, 2005; Olweus, 1993). Investigaciones recientes señalan que la incidencia del maltrato entre iguales en España se sitúa entre el 9.5\% y el 12.5\% (Avilés y Monjas, 2005; Defensor del Pueblo, 2006; Ramírez y Justicia, 2006; Serrano e Iborra, 2005) si bien los índices de prevalencia se mantienen en los últimos años (Defensor del Pueblo, 2006). Tales estudios nos muestran que el maltrato entre alumnos no es un problema nuevo sino que, por el contrario, se conoce desde hace tiempo. En este sentido, se está produciendo una demanda de información y formación por parte del colectivo profesional que más cerca lo vive, el profesorado. Los miembros de este colectivo han tomado conciencia del fenómeno e intentan prevenirlo aunque los resultados no son los deseados (Defensor del Pueblo, 2006). Diversas investigaciones han puesto de manifiesto las quejas del profesorado hacia la falta de formación inicial (Boulton, 1997; Nabuzoka y Smith, 1993; Yoon, 2004). De acuerdo con esta premisa, O’Moore (2000) y Boxer, MusherEizenman, Dubow, Danner y Heretick (2006) señalan la falta de preparación como un gran obstáculo para la prevención y remediación del problema. Además, es importante considerar los hallazgos recientes que señalan las dificultades entre el profesorado para identificar situaciones de maltrato entre iguales (Bauman y Del Rio, 2006; Fernández, García y Benítez, 2006; Hazler, Miller, Carney y Green, 2001) y la creciente necesidad entre el profesorado de conocer el problema, la incidencia, las características, los efectos que causa y cómo combatirlo (Bauman y Del Rio, 2005; Benítez, Berbén y Fernández, 2006; Nicolaides et al. 2002).

Para paliar esta situación, y como una vía de intervención, se hace necesaria la mejora de la formación inicial del profesorado (Benítez et al., 2006) mediante la inclusión de contenidos específicos que ayuden a sensibilizar e informar sobre el fenómeno, a dominar estrategias de intervención, así como a mejorar la capacidad del profesorado para hacer frente los malos tratos (Boulton, 1997; O’Moore, 2000; Yoon, 2004). La formación inicial del profesorado permitiría a los centros educativos contar con personal formado para enfrentar el problema de una forma más global al contar con profesionales que conocen la problemática y están sensibilizados hacia la misma. Los centros educativos podrían establecer políticas edu- 
cativas de centro que considerasen el problema como un desafío colectivo y no como un problema inherente al sistema educativo (UK Observatory of School Violence, 2006).

Benítez, García y Fernández (2007) argumentan la importancia de la formación específica del profesorado: (a) para evitar actuaciones inadecuadas del profesorado; (b) evitar que la víctima pueda sentirse abandonada e indefensa al no haber diagnóstico ni intervención por parte del profesorado; (c) la forma de actuar del profesorado sirve como ejemplo al alumnado y puede darles pistas sobre como intervenir; y, (d) establece formas de intervención que pueden ser replicadas por otros docentes (Lawrence y Green, 2005).

Finalmente, es perentorio que el profesorado sepa identificar los abusos entre el alumnado ya que sin diagnóstico no es posible la evaluación e intervención para prevenir y/o reducir el maltrato entre iguales, más aún cuando varios estudios señalan que el profesorado no valora adecuadamente las tasas de incidencia del fenómeno (O’Moore, 2000) y tiene además dificultades para detectar algunos tipos de maltrato como puede ser el relacional (Benítez, Berbén y Fernández, 2005; Yoon, 2004).

El presente estudio tiene como finalidad valorar el impacto de una formación específica sobre maltrato entre iguales en un grupo de profesores en formación. Así, asumimos la hipótesis de la existencia de cambios significativos entre los elementos del grupo experimental en comparación con los miembros del grupo control.

\section{Método}

\section{Participantes}

La muestra está formada por alumnado de la Universidad de Granada de las especialidades de Magisterio en Educación Infantil, Educación Primaria y alumnado que podrá ejercer como profesorado en Educación Secundaria Obligatoria $(\mathrm{N}=199)$. Los grupos experimental $(n=106)$ y control $(n=93)$ tienen en común la pertenencia a dos asignaturas de Libre Configuración Específica. Una de ellas presenta contenidos sobre el Maltrato entre Iguales (Grupo Experimental) y la otra es ajena a la temática (Grupo Control). Del grupo control se elimina el alumnado que este cursando o haya cursado la asignatura específica sobre Maltrato entre Iguales. La distribución en función de género es similar en ambos grupos: 20,8\% de hombres y $79,2 \%$ de mujeres en el grupo control, y, 20,7\% de hombres y $79,3 \%$ de mujeres en el grupo 
experimental. La edad media de los miembros del grupo control se sitúa en los 20,2 años mientras que en el grupo experimental es de 20,6 años.

\section{Instrumento}

El instrumento utilizado para la recogida de datos es el Cuestionario sobre Maltrato entre Iguales en la Escuela (Nicolaides et al., 2002) traducido y adaptado para esta investigación (Benítez et. al, 2006) (Alpha de Cronbach = 0.941). El cuestionario es anónimo y consta de 45 ítems repartidos en varias secciones que recogen información relativa a:

(a) experiencias personales de malos tratos en la escuela;

(b) conocimiento sobre el fenómeno del maltrato;

(c) conocimiento de las características de víctimas y agresores;

(d) creencias previas acerca del abuso entre iguales;

(e) autocapacidad percibida para afrontar el problema;

(f) estrategias que utilizaría como futuro docente para tratar el fenómeno;

(g) valoración de contenidos de formación específicos.

\section{Diseño y procedimiento}

El diseño de la investigación es de tipo cuasi-experimental de dos grupos no equivalentes con pretest y postest en el que uno de ellos actúa como grupo experimental y otro como grupo control. La cuasi-experimentalidad del estudio viene dada por la no asignación aleatoria de los sujetos a cada uno de los grupos. Los grupos están formados previamente dado que se utiliza como variable de agrupación la pertenencia a una asignatura de Libre Configuración Específica.

La administración del cuestionario se realiza de forma grupal y en horario lectivo previa petición al profesor responsable del grupo. Se establecen dos momentos para la recogida de datos coincidiendo con el inicio (medida pre-tratamiento) y el final (medida posttratamiento) de las asignaturas. El tiempo efectivo de aplicación del instrumento es de 30 minutos.

La formación sobre maltrato entre iguales para los miembros del grupo experimental tiene una duración de 60 horas distribuidas en dos sesiones semanales de dos horas cada una. Los contenidos del curso tienen como finalidad contextualizar la violencia escolar en los centros educativos y presentar del fenómeno de los malos tratos entre iguales: definición y carac- 
terización del problema, factores etiológicos, análisis de los agentes implicados, efectos del maltrato, evaluación del fenómeno y conocimiento y prácticas de intervención para prevenir y remediar el maltrato entre iguales.

Tras la recogida de los cuestionarios los datos se analizan con el paquete estadístico SPSS v. 14.0 realizando análisis de tipo descriptivo, pruebas paramétricas (ANOVA, Bonferroni) y análisis correlacionales en función de la naturaleza de las variables analizadas.

\section{Resultados}

\section{Definición del fenómeno}

El maltrato entre iguales se define, según el instrumento utilizado, atendiendo a las siguientes características: (a) ser un comportamiento intencional; (b) por el carácter físico y/o psicológico; (c) ser frecuente y perdurable en el tiempo; y, (d) la existencia de un desequilibrio de poder entre víctimas y agresores.

La caracterización que los participantes realizan del maltrato entre iguales (Tabla 1) es dispar según el grupo analizado. Los miembros del grupo experimental en la medida postintervención caracterizan el fenómeno atendiendo a un mayor número de características $[F(3)=79.416, p<.001]$ (prueba post-hoc Bonferroni) en tanto que no se observan diferencias significativas entre los miembros del grupo control. El 41.5\% de los miembros del grupo experimental define el maltrato atendiendo al menos a tres características frente a $1.2 \%$ que lo hacía en la evaluación inicial. Se observa una disminución del porcentaje de participantes del grupo experimental que caracteriza el fenómeno atendiendo a una o ninguna de las características definitorias del mismo (del 95.1\% al 21.8\%).

Tabla 1. Grupos y porcentajes de características señaladas para definir el maltrato

\begin{tabular}{lccccc}
\hline & \multicolumn{5}{c}{ Cantidad de características señaladas } \\
\hline Grupo & Ninguna & Una & Dos & Tres & Cuatro \\
& & & & & \\
\hline Control Pre & 40.6 & 48.1 & 11.3 & .0 & .0 \\
\hline Control Post & 38.1 & 46.4 & 13.4 & 1.0 & 1.0 \\
\hline Experimental Pre & 43.2 & 51.9 & 3.7 & 1.2 & .0 \\
\hline Experimental Post & 8.0 & 13.8 & 35.6 & 33.3 & 9.2 \\
\hline
\end{tabular}


El carácter físico y/o psicológico del maltrato entre iguales es la característica que con más frecuencia señalan los participantes independientemente del grupo y momento de medida. En el resto de características aparecen diferencias porcentuales según el grupo analizado (Gráfico 1). Realizada la prueba ANOVA (Bonferroni) se observan diferencias entre los miembros del grupo experimental en la fase post-intervención con el resto dado que señalan con mayor frecuencia: (a) el carácter físico/psicológico $[\mathrm{F}(3)=15.294, \mathrm{p}<.001]$; (b) la intencionalidad $[\mathrm{F}(3)=80.167, \mathrm{p}<.001] ; \mathrm{y}$, (c) la frecuencia y duración $[\mathrm{F}(3)=50.036, \mathrm{p}<.001]$.

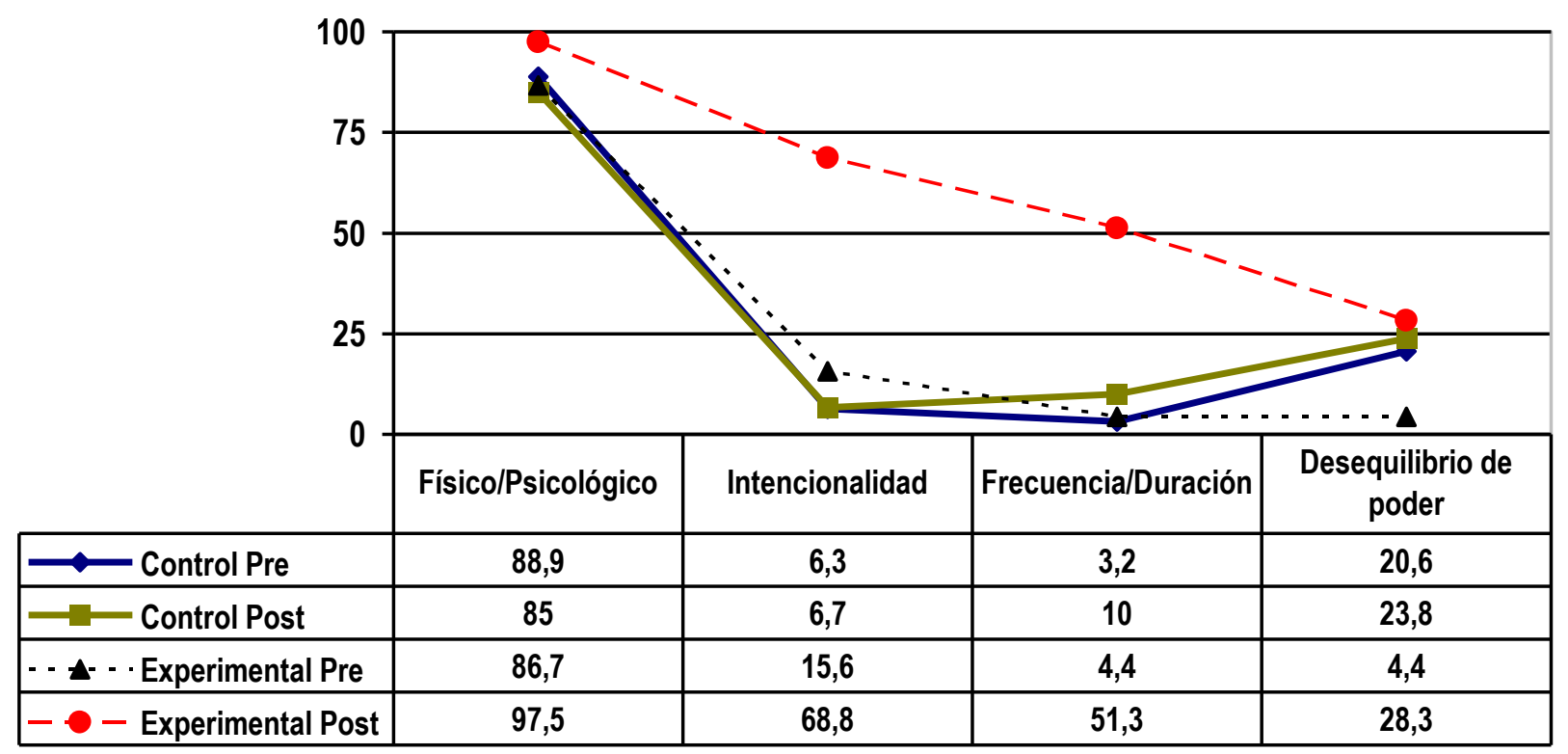

Gráfico 1. Características más señaladas (\%) y grupo

\section{Incidencia del fenómeno}

Para conocer la percepción de los participantes sobre la incidencia del maltrato se les solicita que estimen los porcentajes de alumnos agresores, víctimas y espectadores durante la escolarización (Tabla 2). Se utilizan escalas desde 0 hasta 100 distribuidas en intervalos de 5 puntos.

El análisis de los resultados (ANOVA, prueba post-hoc Bonferroni) muestra diferencias estadísticamente significativas en el caso del grupo experimental no presentando cambios significativos el grupo control. Las diferencias se producen en la percepción del porcentaje de agresores $[F(3)=16.062, p=.000]$ y de víctimas $[F(3)=39.594, p=.000]$. En relación con la estimación del porcentaje de espectadores, encontramos diferencias estadísticamente significati- 
vas entre los miembros de los grupos experimental y control en la medida post-tratamiento $[\mathrm{F}(3)=3.378, \mathrm{p}<.05]$.

Tabla 2. Estimación de porcentajes de agentes implicados en el maltrato y grupos

\begin{tabular}{lccc}
\hline Grupo & Agresores & Víctimas & Espectadores \\
\hline Control Pre & 30.86 & 38.03 & 65.00 \\
\hline Control Post & 31.80 & 41.53 & 63.92 \\
\hline Experimental Pre & 34.88 & 44.00 & 70.37 \\
\hline Experimental Post & 12.95 & 16.15 & 73.05 \\
\hline
\end{tabular}

\section{Características personales de agresores y víctimas}

En el cuestionario se analizan las características personales de agresores y víctimas según las perciben los futuros docentes quienes valoran cada opción en una escala tipo Likert donde $0=$ nunca, 1 = algunas veces y 2 = sí, a menudo. Los participantes en el estudio, independientemente del grupo y momento de la medida, señalan características similares entre el alumnado agresor (Tabla 3). Las más frecuentes son la irritabilidad, la popularidad, las dificultades de aprendizaje, la dificultad para hacer las tareas y el físico fuerte.

En la valoración post-tratamiento los miembros del grupo experimental añaden como características frecuentes la falta de habilidades sociales, el tener amigos que les apoyen, y por último, la falta de ansiedad y preocupación en los agresores. Un análisis de la varianza (prueba post-hoc Bonferroni) señala diferencias estadísticamente significativas al caracterizar a los agresores atendiendo a la irritabilidad $[\mathrm{F}(3)=5.264, \mathrm{p}<.005]$, a la fortaleza física $[F(3)=5.435, p=.005]$, y al tener amigos $[F(3)=7.128, p=.000]$. También se observan diferencias estadísticamente significativas en los miembros del grupo experimental y control (medida post-tratamiento) respecto a que los agresores no hacen bien los deberes, a los niveles de ansiedad y estrés de los mismos, y, a la ausencia de discapacidades físicas en los agresores.

Las características personales de las víctimas del maltrato más señaladas por los participantes son: la debilidad física, la baja autoestima, el tener pocos amigos, la pasividad, la ansiedad, la falta de habilidades sociales y la baja popularidad (Tabla 4). En las medidas post no se detectan diferencias significativas entre los miembros del grupo control, en tanto que se constatan entre los miembros del grupo experimental. Los datos post-tratamiento obtenidos en el grupo experimental asignan mayor importancia a características tales como: (a) la baja 
autoestima $[\mathrm{F}(3)=5.066, \mathrm{p}<.005]$; (b) tener pocos amigos $[\mathrm{F}(3)=3.190, \mathrm{p}<.05]$; (c) preocupación/ansiedad $[\mathrm{F}(3)=22.023, \mathrm{p}=.000] ; \mathrm{y},(\mathrm{d})$ falta de habilidades sociales $[\mathrm{F}(3)=3.338,<.05]$.

Tabla 3. Características personales de los agresores, grupos y diferencias estadísticas

\begin{tabular}{ll|l|l|l|l}
\cline { 2 - 6 } Características Agresores & $\begin{array}{c}\bar{X} \\
\text { GC Pre }\end{array}$ & $\begin{array}{c}\bar{X} \\
\text { GC Post }\end{array}$ & $\begin{array}{c}\bar{X} \\
\text { GE Pre }\end{array}$ & $\begin{array}{c}\bar{X} \\
\text { GE Post }\end{array}$ & Sig. \\
\hline Se irritan fácilmente & 1.75 & 1.72 & 1.45 & $\mathbf{1 . 8 4}$ & .001 \\
Son populares & 1.62 & 1.72 & 1.74 & 1.80 & - \\
Tienen dificultades aprendizaje & 1.47 & 1.53 & 1.57 & 1.57 & - \\
No hacen bien deberes & 1.39 & 1.47 & 1.63 & $\mathbf{1 . 6 3}$ & .007 \\
Físico fuerte & 1.34 & 1.36 & 1.37 & $\mathbf{1 . 6 2}$ & .001 \\
Carecen habilidades sociales & 1.00 & 1.10 & 1.15 & $\mathbf{1 . 3 4}$ & .007 \\
Siempre preocupados y ansiosos & .98 & .99 & .80 & $\mathbf{. 7 2}$ & .003 \\
Tienen baja autoestima & .95 & .98 & 1.10 & .91 & - \\
Tienen pocos amigos & .85 & .80 & .83 & $\mathbf{. 4 5}$ & .000 \\
Son pasivos o poco asertivos & .80 & .74 & .90 & .82 & - \\
Físicamente débiles & .38 & .36 & .29 & .28 & - \\
Tienen discapacidad física & .18 & .29 & .10 & $\mathbf{. 0 6}$ & .000 \\
\hline
\end{tabular}

Tabla 4. Características personales de las víctimas, grupos y diferencias estadísticas

\begin{tabular}{ll|l|l|c|c}
\cline { 2 - 6 } Características Víctimas & $\begin{array}{c}\bar{X} \\
\text { GC Pre }\end{array}$ & $\begin{array}{c}\bar{X} \\
\text { GC Post }\end{array}$ & $\begin{array}{c}\bar{X} \\
\text { GE Pre }\end{array}$ & $\begin{array}{c}\bar{X} \\
\text { GE Post }\end{array}$ & Sig. \\
\hline Físicamente débiles & 1.65 & 1.65 & 1.74 & 1.78 & - \\
Tienen baja autoestima & 1.55 & 1.45 & 1.48 & $\mathbf{1 . 7 7}$ & .002 \\
Tienen pocos amigos & 1.38 & 1.29 & 1.29 & $\mathbf{1 . 5 2}$ & .024 \\
Tienen discapacidad física & 1.31 & 1.26 & 1.37 & 1.38 & - \\
Son pasivos o poco asertivos & 1.22 & 1.21 & 1.30 & 1.29 & - \\
Carecen habilidades sociales & 1.19 & 1.20 & 1.30 & $\mathbf{1 . 6 6}$ & .025 \\
Siempre preocupados y ansiosos & 1.06 & 1.05 & 1.38 & $\mathbf{1 . 6 6}$ & .000 \\
Tienen dificultades aprendizaje & .97 & .87 & .86 & .76 & - \\
No hacen bien deberes & .76 & .66 & .72 & .64 & - \\
Se irritan fácilmente & .45 & .46 & .72 & .61 & - \\
Físico fuerte & .39 & .39 & .32 & .25 & - \\
Son populares & .25 & .22 & .22 & .13 & - \\
\hline
\end{tabular}


Características familiares de agresores y víctimas

Otro aspecto sobre el que opinan los encuestados está relacionado con las características familiares de agresores y víctimas. Los participantes valoran cada opción en una escala tipo Likert donde $0=$ nunca, $1=$ algunas veces y $2=$ sí, a menudo.

En cuanto a las características familiares de los agresores (Tabla 5) los participantes señalan, tanto en el pre-tratamiento como en el post-tratamiento, las mismas características: disciplina inconsistente, abuso emocional o físico, relaciones distantes y castigo físico. Aparecen diferencias entre los miembros el grupo experimental quienes les conceden mayor importancia en la medida post-intervención, siendo estadísticamente significativa la valoración de las relaciones interpersonales distantes $[F(3)=9.656, p=.000]$ y a la disciplina inconsistente $[F(3)=3.276, p=.05]$.

Tabla 5. Características familiares de los agresores, grupo y diferencias significativas.

\begin{tabular}{ll|l|l|l|l}
\cline { 2 - 6 } Características Familiares & \multicolumn{1}{c|}{$\begin{array}{c}\bar{X} \\
\text { GC Pre }\end{array}$} & $\begin{array}{c}\bar{X} \\
\text { GC Post }\end{array}$ & $\begin{array}{c}\bar{X} \\
\text { GE Pre }\end{array}$ & $\begin{array}{c}\bar{X} \\
\text { GE Post }\end{array}$ & Sig. \\
\hline Disciplina inconsistente & 1.36 & 1.33 & 1.49 & $\mathbf{1 . 5 8}$ & .015 \\
Abuso emocional o físico & 1.29 & 1.37 & 1.36 & 1.40 & - \\
Relaciones Inter. Distantes & 1.28 & 1.30 & 1.34 & $\mathbf{1 . 6 7}$ & .000 \\
Castigo físico & 1.26 & 1.33 & 1.39 & 1.42 & - \\
Sobreprotegidos & .84 & .99 & .78 & .78 & - \\
Hogar normal & .81 & .81 & .73 & .74 & - \\
Relaciones Inter. Positivas & .46 & .52 & .41 & .36 & - \\
\hline
\end{tabular}

El análisis de las características familiares de las víctimas (Tabla 6) permite observar cómo ambos grupos señalan las mismas tanto en la evaluación inicial como en la final.

Sin embargo, se observan diferencias estadísticamente significativas entre las respuestas del grupo experimental quienes, en la evaluación post-intervención, señalan como más frecuentes la sobreprotección $[F(3)=6.768, p=.000]$, las relaciones distantes $[F(3)=12.606$, $\mathrm{p}=.000]$, y las relaciones positivas $[\mathrm{F}(3)=4.173, \mathrm{p}<.01]$. 
Tabla 6. Características familiares de las víctimas, grupo y diferencias significativas.

\begin{tabular}{ll|l|l|c|c}
\cline { 2 - 6 } Características Familiares & \multicolumn{1}{c|}{$\begin{array}{c}\bar{X} \\
\text { GC Pre }\end{array}$} & $\begin{array}{c}\bar{X} \\
\text { GC Post }\end{array}$ & $\begin{array}{c}\bar{X} \\
\text { GE Pre }\end{array}$ & $\begin{array}{c}\bar{X} \\
\text { GE Post }\end{array}$ & Sig. \\
\hline Sobreprotegidos & 1.45 & 1.46 & 1.52 & $\mathbf{1 . 7 7}$ & .000 \\
Hogar normal & 1.23 & 1.27 & 1.30 & 1.19 & - \\
Rel. Interp. Distantes & 0.79 & 1.04 & .69 & $\mathbf{1 . 1 7}$ & .000 \\
Rel. Interp. Positivas & 1.04 & 1.10 & 1.26 & $\mathbf{1 . 3 2}$ & .006 \\
Abuso emocional o físico & .98 & .87 & .75 & .98 & - \\
Disciplina inconsistente & .74 & .75 & .57 & .55 & - \\
Castigo físico & .57 & .54 & .47 & .53 & - \\
\hline
\end{tabular}

Auto-eficacia para enfrentar el maltrato entre iguales

El último apartado valorado se relaciona con la percepción sobre la capacidad propia para llevar a cabo acciones destinadas a tratar el problema. La auto-eficacia percibida por los miembros del grupo control no presenta cambios significativos, en tanto en cuanto, el grupo experimental presenta cambios estadísticamente significativos en relación con el trato directo con los agentes implicados y con el trato con las familias (Tabla 7).

Tabla 7. Diferencias estadísticas y percepción sobre la capacidad personal

\begin{tabular}{lcccccc}
\cline { 2 - 7 } & & & & Dif. $\bar{X}$ GE & Error & \\
& g.l. & F & Sig. & Post / Pre & Típico & Sig. \\
\hline Hablar con agresores sin culpar & 3 & 142.324 & .000 & $1.410\left(^{*}\right)$ & .135 & $<.001$ \\
Hacer que agresores paren & 3 & 293.724 & .000 & $.880\left(^{*}\right)$ & .104 & $<.001$ \\
Hablar con víctimas sin culpar & 3 & 683.356 & .000 & $1.107\left(^{*}\right)$ & .078 & $<.001$ \\
Apoyar a las víctimas & 3 & 1210.467 & .000 & $1.032\left(^{*}\right)$ & .062 & $<.001$ \\
Hablar con espectadores & 3 & 867.353 & .000 & $.980\left(^{*}\right)$ & .073 & $<.001$ \\
Formar espectadores para ayudar & 3 & 618.846 & .000 & $1.093\left(^{*}\right)$ & .086 & $<.001$ \\
Trabajar con los padres de víctimas & 3 & 577.084 & .000 & $.974(*)$ & .088 & $<.001$ \\
Trabajar con los padres de agresores & 3 & 501.768 & .000 & $.952\left(^{*}\right)$ & .090 & $<.001$ \\
\hline
\end{tabular}

* La diferencia de medias es significativa al nivel .05

\section{Discusión y conclusiones}

La revisión de los resultados muestra el impacto positivo que sobre los sujetos del grupo experimental ha tenido el curso de formación sobre malos tratos entre iguales. En este 
sentido se han encontrado diferencias estadísticamente significativas en cuanto al conocimiento del fenómeno.

Un primer dato de cambio lo obtenemos cuando revisamos la capacidad de los participantes para definir el problema. Diversas investigaciones han puesto de manifiesto la incapacidad del profesorado para definir el fenómeno de manera adecuada (Bauman y Del Río, 2006; Fernández, et al., 2006; Hazler et al., 2001; Yoon 2004) y así queda reflejado en nuestro estudio, si revisamos los valores de la medida inicial en grupo experimental y control a este respecto. No obstante, tras el curso de formación, la capacidad para definir el problema ha mejorado significativamente entre los miembros del grupo experimental. Éstos son capaces de definir el maltrato de forma más certera y basándose en más características definitorias del mismo para hacerlo.

A la hora de definir el fenómeno, observamos cómo los participantes no tienen dificultades para definir el maltrato como un conjunto de comportamientos de carácter físico y/o psicológico. Sin embargo, características tales como el desequilibrio de poder, la intencionalidad, la frecuencia y la duración no se toman en consideración. Estos resultados obtenidos en la medida inicial son similares a los obtenidos en otras investigaciones que apuntan en la misma dirección (Benítez et al., 2006; Boulton, 1997; Hazler et al., 2001; O’Moore, 2000; Siann, Callaghan, Lockhart y Rawson, 1993). Sin embargo, los datos varían al revisar los resultados finales del grupo experimental. Los sujetos del grupo experimental consideran no sólo la característica físico-psicológica sino que también incluyen en la definición la intencionalidad del comportamiento abusivo, la recurrencia y duración del fenómeno y la diferencia de poder entre víctima y agresor.

La misma situación encontramos si analizamos las estimaciones del número de agresores, víctimas y espectadores realizadas por los miembros de ambos grupos. Nicolaides et al. (2002) y Benítez et al. (2006) señalan que el profesorado tiende a sobreestimar el número de agentes implicados en el maltrato entre iguales. En el presente trabajo, este dato se confirma en la evaluación pre-tratamiento mientras que en la post-tratamiento se observan diferencias significativas entre los miembros del grupo experimental. Éstos últimos sobreestiman ligeramente los porcentajes de agentes implicados (12,95\% de agresores, 16,15\% de víctimas y $73,05 \%$ de espectadores) aunque se aproximan más a los datos reales obtenidos por investiga- 
ciones sobre incidencia (Avilés y Monjas, 2005; Smith, Morita, Junger-Tas, Olweus, Catalana y Slee; 1999) y son significativamente diferentes a los obtenidos en la medida inicial.

Una revisión de trabajos sobre maltrato entre iguales nos permite caracterizar al agresor por el fuerte temperamento e irritabilidad, por una autoestima normal (Smith et al., 1999; Smith, 2004), por su poder físico (Card, 2003), por poseer unas habilidades sociales precarias (Kaukiainen, Björkqvist, Lagerspetz, Österman, Salmivalli, Forsblom y Ahlbom, 1999). Los participantes en el estudio, en la misma línea que otros (Benítez et al., 2006), señalan la irritabilidad, la popularidad, las dificultades de aprendizaje, la dificultad para hacer las tareas y el físico fuerte, como las características más frecuentes entre los agresores. Sin embargo, y sólo en el caso del grupo experimental, en la evaluación post-tratamiento observamos otras características tales como la falta de habilidades sociales, el tener amigos que les apoyen, y por último, la falta de ansiedad y preocupación en los agresores. De nuevo, encontramos cambios significativos dado que los sujetos que reciben la formación concretizan mejor las características de los agresores.

Conclusiones similares obtenemos cuando revisamos los datos sobre las características personales de las víctimas. Inicialmente caracterizan a la víctima por la debilidad física (Smith et al., 1999), la baja autoestima (Donnellan, Trzesniewski, Robins, Moffitt y Caspi, 2005), el tener pocos amigos y la baja popularidad (Laukkanen, Shemeikka, Notkola, Koivumaa-Honkanen y Nissinen, 2002), la pasividad, el estado de ansiedad y la falta de habilidades sociales (Fox y Boulton, 2005). Sin embargo, y tras la formación específica, son más los sujetos del grupo experimental que señalan tales características entre el alumnado víctima. Siendo significativa la mayor importancia que estos conceden a la baja autoestima de la víctima, a tener pocos amigos, al estado de preocupación/ansiedad y, la falta de habilidades sociales como características más frecuentes entre las víctimas.

En la misma dirección que en los casos anteriores nos encontramos cuando analizamos las características familiares de agresores y víctimas. Es decir, un conocimiento más exacto ente los miembros del grupo experimental, a diferencia de sus creencias iniciales y de las señaladas por los miembros del grupo control.

Para finalizar, es necesario destacar el cambio producido en la percepción de los sujetos del grupo experimental sobre la autocapacidad para afrontar el maltrato entre iguales. El cambio es significativo en todas y cada una de las opciones. Se autoperciben como más capa- 
ces para tratar directamente con víctimas y agresores, así como para tratar con los padres de los mismos, o para trabajar con los espectadores para prevenir y/o remediar el maltrato entre iguales.

Los resultados obtenidos permiten afirmar que los miembros del grupo experimental han modificado y mejorado los conocimientos y percepciones sobre el maltrato entre iguales, así como que se perciben como más capaces para poder afrontarlo. Observamos como una formación específica sobre el maltrato entre iguales tiene un impacto positivo y permite conocer más profundamente el problema. Estos resultados podrían llevarnos a discutir sobre la necesidad de introducir contenidos específicos, no sólo relacionados con el maltrato entre iguales sino con otros problemas educativos, en los planes de estudio del profesorado que permitan al alumnado conocer problemas educativos reales que tendrán que enfrentar en el futuro, y con qué mejor aval, que el saber cómo combatirlos.

\section{Referencias}

Avilés, J. M. y Monjas, I. (2005). Estudio de la incidencia de la intimidación y el maltrato entre iguales en la educación secundaria obligatoria mediante el cuestionario CIMEI (Avilés, 1999) - Cuestionario sobre Intimidación y Maltrato Entre Iguales -. Anales de Psicología, 21(1), 27-41.

Bauman, S. y Del Rio, A. (2005). Knowledge and beliefs about bullying in schools: comparing pre-service teachers in United Status and the United Kingdom. School Psychology International, 26(4), 428-442.

Bauman, S. y Del Rio, A. (2006). Preservice Teachers' Responses to Bullying: Comparing Physical, Verbal, and Relational Bullying. Journal of Educational Psychology, 98(1), 219-231.

Benítez, J. L., Berbén, A. G. y Fernández, Ma (2005). Conocimiento y actitud del maltrato entre alumnos (bullying) de los futuros docentes de Educación Infantil, Primaria y Secundaria. Revista de Enseñanza Universitaria, 26, 71-84.

Benítez, J. L., Berbén, A. G. y Fernández, Mª (2006). El maltrato entre alumnos: conocimientos, percepciones y actitudes de los futuros docentes. Revista de Investigación Educativa, 24(2), 329-352

Benítez, J. L.; García, A. y Fernández, Mª (2007). Eficacia de un curso de formación sobre el maltrato entre iguales en el currículum inicial del profesorado. En J. J. Gázquez, Ma C. 
Pérez, A. Cangas y N. Yuste (Coords.) Mejora de la convivencia y programas encaminados a la prevención e intervención del acoso escolar. Granada. Grupo Editorial Universitario.

Boulton, M. J. (1997). Teachers' views on bullying definitions, attitudes and ability to cope. British Journal of Educational Psychology, 67, 223-233.

Boxer, P., Musher-Eizenman, D., Dubow, E. F., Danner, S. y Heretick, A. D. (2006). Assessing teachers' perceptions for school-based aggression prevention programs: Applying a cognitive-ecological framework. Psychology in the Schools, 43(3), 331-344.

Card, N. A. (2003). Victims of peer aggression: A meta-analytic review. Presented at Society for Research in Child Development biennial meeting, Tampa, USA.

Cerezo, F. (2006). Violencia y victimización entre escolares. El Bullying: estrategias de identificación y elementos para la intervención a través del test Bull-s. Electronic Journal of Research in Educational Psychology, 9,4(2), 333-352.

Defensor del Pueblo (2006). Violencia Escolar:el maltrato entre iguales en la Educación Secundaria Obligatoria 1999-2006. Recuperado el 15/11/2007, de http://www.defensordelpueblo.es/documentacion/informesmonograficos/ViolenciaEsc olar2006.pdf

Del Barrio, C., Gutiérrez, H., Barrios, A., Van der Meulen, K. y Granizo, L. (2005). Maltrato por abuso de poder entre escolares, ¿de qué estamos hablando? Revista de Pediatría y Atención Primaria, 7, 75-100.

Donnellan, M. B., Trzesniewski, K. H., Robins, R. W., Moffitt, T. E., y Caspi, A. (2005). Low self-esteem is related to aggression, antisocial behavior and delinquency. Psychological Science, 16(4), 328-335.

Fernández, Mª ., García, A. y Benítez, J. L. (2006). Estudio de la percepción que el profesorado en activo posee sobre el maltrato entre iguales. Profesorado. Revista del currículum y formación del profesorado, 10(2).

Fox, C. y Boulton, M. (2005). The social skills problems of victims of bullying: self, peer and teacher perceptions. British Journal of Educational Psychology 75, 313-328.

Hazler, R., Miller, D. L., Carney, V. y Green, S. (2001). Adult recognition of bullying situations. Educational Research, 43(2), 133-146. 
Kaukiainen, A., Björkqvist, K., Lagerspetz, K., Österman, K., Salmivalli, C., Forsblom, S., y Ahlbom, A. (1999). The relationships between social intelligence, empathy, and three types of aggression. Aggressive Behavior, 25, 81-89.

Laukkanen, L., Shemeikka, S., Notkola, I., Koivumaa-Honkanen, H. y Nissinen, A. (2002). Externalizing and internalizing problems at school as signs of health-damaging behavior and incipient marginalization. Health Promotion International, 17(2), 139-146.

Lawrence, C. y Green, K. (2005). Perceiving classroom aggression: the influence of setting, intervention style and group perceptions. British Journal of Educational Psychology, $75,587-602$.

Nabuzoka, D. y Smith, P. K. (1993). Sociometric status and social behaviour of children with and without learning difficulties. Journal of Child Psychology and Psychiatry, 34, $1435-1448$

Nicolaides, S., Toda, Y., y Smith, P. K. (2002). Knowledge and attitudes about school bullying in trainee teachers. British Journal of Educational Psychology, 72, 105-118.

Olweus, D. (1993). Bullying at school: What we know and what we can do. Oxford, UK: Blackwell.

O’Moore, M. (2000). Critical issues for teacher training to counter bullying and victimisation in schools. Aggressive Behavior, 26, 99-111.

Ramírez, S. y Justicia, F. (2006). El maltrato entre escolares y otras conductas problema para la convivencia. Electronic Journal of Research in Educational Psychology, 4(2), 265289.

Serrano, A. e Iborra, I. (2005). Violencia entre compañeros en la escuela. Centro Reina Sofía para el Estudio de la Violencia. Valencia: Goeprint.

Siann, G., Callaghan, M., Lockhart, R. y Rawson, L. (1993). Bullying: teachers' views and school effects. Educational Studies, 19, 307-321.

Smith, P. K. (2004). Bullying: Recent Developments. Child and Adolescent Mental Health 9(3), 98-103.

Smith, P.K., Morita,Y., Junger-Tas, Olweus, D., Catalano, R. y Slee, P.T. (Eds) (1999). The Nature of School Bullying: a Cross-National Perspective. London: Routledge.

UK Observatory Of School Violence (2006). Violence in School Training Action. Recuperado el 15/06/2007, de http://www.vista-europe.org/downloads/VISTA_Introduction.pdf

Yoon, J. (2004). Predicting teacher interventions in bullying situations. Education and Treatment of Children, 27(1), 37-45. 
Juan L. Benítez et al.

[Página en blanco por razones de paginación] 